\title{
Politik als Beruf: die nächste Generation
}

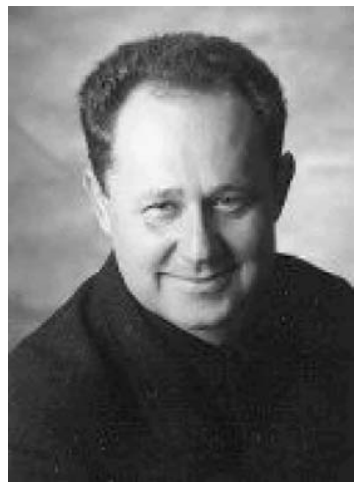

Prof. Dr. M. Wildner

Bibliografie

DOI http://dx.doi.org/

10.1055/s-0031-1298007

Gesundheitswesen 2011;

73: 789-790

(c) Georg Thieme Verlag KC

Stuttgart · New York

ISSN 0941-3790

Korrespondenzadresse

Prof. Dr. M. Wildner

Bayerisches Landesamt für

Gesundheit und Lebens-

mittelsicherheit

Veterinärstraße 2

85762 Oberschleißheim

manfred.wildner@|gl.bayern.de
„Einen gegebenen Menschen in die richtige Verfassung zu bringen ist nicht etwas für den Erstbesten, sondern wenn überhaupt für den Wissenden“, räsoniert Aristoteles im vierten vorchristlichen Jahrhundert [1]. Und er fährt fort: „In den anderen Disziplinen sind offenkundig diejenigen, welche die Fähigkeiten weitergeben, und diejenigen, welche sie ausüben, dieselben, wie bei Ärzten und Malern. Was hingegen die politischen Dinge betrifft, so sind es die Sophisten, die sie zu lehren beanspruchen, doch keiner von ihnen übt sie aus“. Und etwas hintergründig fügt er hinzu: „Dies tun vielmehr die Politiker, die es, so könnte man denken, eher aufgrund einer bestimmten Anlage oder Erfahrung als aufgrund von Denken tun!“.

Positiv verstanden wird gute Politik damit vor allem mit Intuition und wohlverstandenem Bauchbzw. Fingerspitzengefühl verbunden. Für ein zeitgemäßes rationales Politikverständnis stellt sich damit die Frage: Kann Politik unterrichtet werden? Noch wichtiger: kann Politik erlernt werden? „Yes, we can!“ (dt.: „Wir schaffen das schon!“), mag man/frau versucht sein, zukunftsoptimistisch schnell zu antworten. Mit etwas Achtsamkeit und Bedachtsamkeit sind auch andere Stimmen zu erwägen, z.B. die eines Altkanzlers und Publizisten: „Wer mit 18 Jahren Politiker werden möchte, der kann mir gestohlen bleiben. Er soll gefälligst einen Beruf lernen und diesen Beruf ausgeübt haben, mit Erfolg ausgeübt haben. Danach kann er sich anschließend gerne politisch engagieren“ [2]. Die Politiksoziologie bestätigt die implizierte Vermutung: Politiker-Biografien insbesondere der jüngeren Generation weisen den erlernten Beruf häufig nur mehr als Beiwerk auf. Stattdessen finden sich Karrieren über die Kaderschmieden der jeweiligen politischen Jugendorganisationen. Karrieren fernab der Sorgen und Nöte der Menschen im Land, dafür erprobt in nächtelangen Diskussionen und politischen Kompromissen [3]?

Mit der Frage von „Politik als Beruf“ hat sich Max Weber im Rahmen eines Vortrages vor dem Münchner Freistudentischen Bund im Januar 1919 befasst [4]. „Wer Politik treibt, erstrebt Macht“, hält Weber nüchtern fest. Dabei seien drei Qualitäten für den Berufspolitiker entscheidend: Leidenschaft im Sinne des leidenschaftlichen Dienstes an einer Sache, Verantwortungsgefühl und Augenmaß. Diese haben ihre jeweils eigenen Zeithorizonte. Verantwortlichkeit bedeutet nicht nur, „die Sache“ fachlich zu bearbeiten, sondern auch, sich die damit verbundenen Handlungen zum persönlichen Anliegen zu machen und Verantwortung für die Folgen zu übernehmen. Augenmaß soll heißen „die Fähigkeit, die Realitäten mit innerer Sammlung und Ruhe auf sich wirken zu lassen, also: der Distanz zu den Dingen und Menschen“. Max Weber sieht die „Sünde gegen den heiligen Geist“ in der Politik da beginnen, wo das Machtstreben unsachlich und Gegenstand „reiner Selbstberauschung“ wird, wo nach „Wirkung“ und „Eindruck“ gefragt wird. „Politik bedeutet ein starkes langsames Bohren von harten Brettern mit Leidenschaft und Augenmaß zugleich“ [ebd, S. 82]. „Nur wer sicher ist, dass er daran nicht zerbricht, wenn die Welt, von seinem Standpunkt aus, zu dumm oder zu gemein ist für das, was er ihr bieten will, dass er all dem gegenüber: ,dennoch!' zu sagen vermag, nur der hat den ,Beruf' zur Politik“ [ebd. S. 83].

Nachdenklich nimmt man diese Maßstäbe zur (Selbst-Er)Kenntnis, eingebunden als Bürger, Funktionsträger oder politisch Verantwortlicher in eine allgegenwärtige Medienpräsenz. Ein nicht ganz ernst gemeinter Versuch für einen politischen Gewissensspiegel: Wie steht es um meine Distanz zum Pressespiegel? Wie oft habe ich am Morgen danach die „Schneewittchen“-Frage des „Und, wie war ich?“ gestellt? Und mich damit als zweifelhaft selbstverliebte Königin bzw. (über-) ehrgeiziger Kronprinz erwiesen? Bohre ich noch politisch harte Bretter mit Augenmaß und sachlicher Leidenschaft? Habe ich die Kraft zum „dennoch!"? Fragen, die gerne auch in der rheinischen Bütt aufgegriffen werden, um später weiter südlich in den Fastenpredigten des Nockherbergs vertieft zu werden. Dann allerdings fremdbestimmt: Es darf gelacht werden. Die närrische Zeit als schmerzhafte politische Bußzeit? Alternativ bietet sich ein eigenbestimmtes Resümieren an, z.B. in probater (Buch-)Form der „Bekenntnisse“ [5].

Doch ist der Politiker des 21. Jahrhunderts nicht schon einer nächsten Generation zugehörig, weniger Objekt von Komödien als vielmehr Protagonist einer Tragödie: zwischen zahlreichen Terminen einerseits gehetzt von den schattenhaften Erinyen einer freien Presse, andererseits gezogen vom sirenenhaften Lockruf medialer Präsenz? Verurteilt zum Multitasking in medialen Rollen: als Staatsmann/frau, Schauspieler/in, Chansonnier/ière - politische Spielform des „flexiblen Menschen“ [6]? Lernen lässt sich eine reife Haltung für dieses vielfältige und enorm fordernde „Ausgesetztsein“ in der Politik nur schwer und wohl noch am ehesten in der oben eingeforderten nüchternen Schule eines Brotberufes. 
Gleichzeitig tut für uns als „Zuschauer“ Bescheidenheit Not. All zu gerne lehnen wir uns in unseren Sitzen zurück und vergessen, dass dort auf der Bühne „unsere“ Schauspieler ihre Rolle spielen, dass in dem, was bisweilen wie eine Komödie wirkt, ganz real auch unser Schicksal verhandelt wird. Dass die Akteure einer demokratischen Bühne elementar auf uns angewiesen sind: auf unseren beratenden Sachverstand, unseren Beifall und unsere Kritik und ganz besonders auf die sachverständige Kritik guter Pressearbeit. Deren Wert kann nicht hoch genug eingeschätzt werden. Modernes politisches Handeln ist Kommunikation.

Bisweilen setzt schon unsere Sprache unserem Denken Grenzen. Was im Deutschen unter „Politik“ subsumiert wird, wird im Englischen differenzierter betrachtet und bezeichnet: dort werden politische Inhalte und Strategien (policy), politische Verfahren und Taktiken (politics) und politische Institutionen (polity) sprachlich unterschieden. Strategien haben ihre Zeit, Taktik hat ihre Zeit, und politische Institutionen haben ihren Ort. Manche "Lacher“ würden sich bei einer differenzierteren Analyse der Geschehnisse auf der Bühne erübrigen und als Kategorienfehler auf Zuschauerseite entpuppen: als institutionsblinde, fehlerhafte Verortung politischen Handelns oder als fehlerhafte Einordnung von Taktik als Strategie. Und sich so in verständigeres und damit sachgerechteres Nachdenken wandeln.

Ist mit diesen Skizzen das für das 21. Jahrhundert und auch für die Gesundheitspolitik Wesentliche schon gesagt? Um noch einmal auf Aristoteles' hintergründige Bemerkung zurückzukommen, dass sich politisches Handeln eher aufgrund einer bestimmten Anlage oder Erfahrung als aufgrund von Denken vollzieht: Hier hat sich der Anspruch gewandelt. Politik ist stärker als früher „rechenschaftspflichtig“ geworden. „Accountability“ lautet der Fachausdruck in den einschlägigen europäischen Dokumenten [7].

Diese Rechenschaftspflicht ist in der europäischen Gesundheitspolitik einerseits an konsentierte Werte wie Chancengleichheit, Transparenz und Angemessenheit geknüpft, andererseits auch an „technisches“ Fachwissen. Die Bismarcksche Aussage „Die Politik ist keine Wissenschaft, wie viele der Herren Professoren sich einbilden, sie ist eben eine Kunst" trifft heute nur mehr in Teilaspekten zu. Gesundheitspolitisch relevante Entscheidungen werden vermehrt auf Basis der „besten verfügbaren Evidenz“ gefällt. Dieser Evidenzbegriff wurde ursprünglich im klinischen Bereich als „Evidenzbasierte Medizin (EbM)“ von der Gruppe um den Allgemeinmediziner David Sackett entwickelt [8]. Darunter wird eine wissenschaftlich systematische, explizite und erschöpfende Berücksichtigung des jeweils besten gegenwärtig verfügbaren Wissens zur Beantwortung einer Fragestellung verstanden. Dieser Ansatz konsequent empirisch untermauerter Praxis setzt sich trotz mancher Widerstände im Gesundheitswesen zunehmend durch, auch mit Unterstützung durch den Gesetzgeber. Inzwischen sind davon zentrale gesundheitspolitische Entscheidungsebenen im Sinne einer „Evidenzbasierten Gesundheitsversorgung“ erreicht: Gemeinsamer Bundesausschuss (G-BA), Ärztliche Zentralstelle für Qualitätssicherung (ÄZQ) und das Institut für Qualität und Wirtschaftlichkeit im Gesundheitswesen (IQWiG) seien als Beispiele genannt. Der Umgang mit Evidenz wiederum kann erlernt werden: als methodische Kompetenz für Instrumente wie Health Impact Assessment (HIA), Health Technology Assessment (HTA) oder systematische Evidenzsynthesen zur politischen Entscheidungsunterstützung [9].
Solchen Evidenzen gehen die Beiträge in diesem Heft nach: zur Lebensqualität in Pflegeheimen, zur Qualitätssicherung in Krankenhäusern am Beispiel Dekubitus, zur Überwachung der Krankenhaushygiene, zum Case-Management von Brustkrebspatientinnen, zu einem ganzheitlichem betrieblichen Gesundheitsmanagement, zum Stresserleben in Deutschland, zur Akzeptanz der elektronischen Gesundheitskarte, zur sozialmedizinischen Lehre an den medizinischen Fakultäten und im internationalen Rahmen zum Vergleich der deutschen und italienischen DRGSysteme am Beispiel der Handchirurgie, zur EU-Trinkwasserrichtlinie sowie zur zahnmedizinischen Grundversorgung in Westafrika. Zur Diskussion stellen sich auch ein konzeptioneller Beitrag des Deutschen Netzwerkes Versorgungsforschung zu einer „Projektdatenbank Versorgungsforschung“ sowie der Methodenworkshop „Directed Acyclic Graphs“ der Bundesanstalt für Arbeitsschutz und Arbeitsmedizin (BAuA).

Evidenzbasierte Medizin als Modell einer Evidenzbasierten Gesundheitspolitik? Um Missverständnissen vorzubeugen: Evidenz soll wissensbasiert Entscheidungen unterstützen, nicht die werteorientierte politische Entscheidungsfindung - womöglich in missbräuchlicher Alibifunktion - ersetzen. Bei aller methodischer Kompetenz und Aufbereitung der Evidenz darf und soll um Wertentscheidungen gestritten werden. Vielleicht waren diese Ansätze expliziter Werteorientierung und methodischer Kompetenz auch schon in den 3 Weberschen Qualitäten Leidenschaft, Verantwortung und Augenma $\beta$ vorgedacht. In ihrer modernen wissensbasierten Ausgestaltung sind sie ein innovativer Beitrag des 21. Jahrhunderts. Werteorientiertes, medial transparentes, fachlich ernsthaftes „starkes langsames Bohren von harten Brettern“ als Anspruch an Politiker wie an die Wissensgesellschaft gleichermaßen: spezifische und nachhaltige Schritte auf dem Weg der Professionalisierung von (Gesundheits-)Politik der „nächsten Generation“ im besten Sinne - mit dem eingangs genannten gesamtgesellschaftlichen Ziel, auch uns „gegebene“ Menschen der nächsten Generation in die richtige Verfassung zu bringen.

\section{Literatur}

1 Aristoteles. Nikomachische Ethik. Zehntes Buch: Rowohlt, Hamburg: 2006 S. 340

2 Schmidt $H$. „Seien Sie ernsthaft und fleißig!“. In:, Die Zeit, Hrsg. Helmut Schmidt. Würdigungen, Essays und Gückwünsche zum 90. Geburtstag. Erster Teil: Der Staatsmann. Die Zeit; Hamburg: 2008

3 Nachtwey 0 . In der Mitte gähnt der Abgrund. Blätter für deutsche und internationale Politik 2008; 8: 58-68 http://www.blaetter.de/artikel. php?pr $=2884$ (Zugriff 01.01.2009)

4 Weber M. Politik als Beruf. Reclam; Stuttgart: 1992

5 Sarkozy N. Bekenntnisse. Frankreich, Europa und die Welt im 21. Jahrhundert. C Bertelsmann; München: 2007

6 Sennett $R$. Der flexible Mensch. Bvt Berliner Taschenbuch Verlag; Berlin: 2006 (Engl. Originaltitel: The Corrosion of Character: The Personal Consequences of Work in the New Capitalism. W. W. Norton, 1998)

7 Europäische Gesundheitsministerkonferenz der Weltgesundheitsorganisation Europa. Die Charta von Tallinn: Gesundheitssysteme für Gesundheit und Wohlstand. Tallinn, Estonia, 27. Juni 2008 ( http://www. euro.who.int/document/e91438g.pdf, Zugriff am 02.01.2009)

8 Sackett DL, Rosenberg WM, Gray JA, Haynes RB, Richardson WS. Evidence based medicine: what it is and what it isn't. BMJ 1996; 312: 71-72

9 Ståhl T, Wismar M, Ollila E et al., Hrsg. Health in all policies. Prospects and potentials. Ministry of Social Affairs and Health, Helsinki (Finnland): 2006 